\title{
A New Differential Positioning Method using Modulation Correlation of Signals of Opportunity
}

\author{
Toby A Webb, Paul D Groves, Paul A Cross \\ Dept. Civil, Environmental and Geomatic Engineering \\ University College London \\ London, U.K. \\ toby.webb@ucl.ac.uk
}

\author{
Robert J Mason, Joseph H Harrison \\ Terrafix Ltd. \\ Stoke-on-Trent, \\ Staffordshire, U.K \\ rjm@terrafix.co.uk
}

\begin{abstract}
Global Navigation Satellite Systems (GNSS) have become the positioning systems of choice for many applications. However, GNSS signals are susceptible to obstruction, interference and jamming. Therefore, to maximize robustness and integrity, it is necessary to employ a dissimilar positioning technology that can operate independently and back-up GNSS. One such technology exploits 'signals of opportunity' - signals that are designed for purposes other than navigation.
\end{abstract}

This paper presents a system that uses amplitude modulation (AM) radio broadcasts in the medium frequency (MF) band. At these frequencies, the predominant ground-wave propagation mode offers better coverage in remote areas and over sea than is achievable with higher frequency signals.

The system is differential and operates by correlating modulation information between the reference and user receivers. A system of this form mitigates the deterioration in and around buildings encountered in prior systems and can provide absolute position using fewer signals than a system using only carrier phase.

The system presented in this paper uses generalized cross correlation to obtain time difference of arrival measurements that are subsequently used for position determination. Preliminary results indicate the system provides a robust position solution. Moreover, the system offers the potential to be combined with carrier phase measurements to achieve enhanced performance, while the modulation correlation technique is readily extendable to other types of signal.

Keywords-Signals of Opportunity; Correlation; Amplitude Modulation;

\section{INTRODUCTION}

Global Navigation Satellite Systems (GNSS) have evolved to be the positioning system of choice for many applications. However GNSS signals are susceptible to obstruction, interference and jamming. Signal obstruction is a particular problem indoors, underground and in deep urban canyons. Sources of unintentional interference include adjacent-band satellite communications, harmonics of television transmissions and remote locking devices, radar and terrestrial aircraft navigation signals [1].

The work presented in this paper has been conducted under a Councils Co-Operative Awards in Science and Engineering (CASE) supported by Engineering and Physical Science Research Council (EPSRC) and Terrafix Ltd.
Deliberate GNSS jamming has historically been a military issue. However the introduction of GNSS-based law enforcement, high-value asset tracking and, potentially, road user charging have made jamming of interest to criminals. Low-cost jammers, covering all GNSS bands, are now easily obtainable via the internet. Although most of these jammers have a very short range, some can disrupt GNSS reception over tens of kilometres [2].

A further source of GNSS disruption is space weather. During solar maxima, ionospheric scintillation can disrupt reception of most GNSS signals in polar and equatorial regions at certain times of day [3], while a solar flare can knock out all GNSS reception over the side of the Earth facing the sun [4].

Therefore, to maximise the robustness of a position solution, in terms of availability, continuity and integrity, it is necessary to augment GNSS with one or more dissimilar positioning technologies. These technologies must operate independently of GNSS and be subject to different failure modes.

Navigation and positioning technologies may be divided into two categories: dead reckoning and position fixing [5]. Dead-reckoning technologies measure the distance travelled and direction. Examples include inertial navigation, odometers, magnetometers and Doppler radar and sonar. Positioning using dead reckoning requires a known starting point and the errors are cumulative, so the position error grows with time. However, there is no reliance on landmarks.

In position fixing, the user position is determined by measuring the range and/or direction to landmarks at known locations (or by being in close proximity to the landmark). Thus, for a position solution to be obtained, sufficient known landmarks must be available. However, there is no degradation in accuracy with time. Position fixing techniques may be divided into two further categories: radio navigation and feature matching [5]. In radio navigation systems, the radio transmitters normally constitute the landmarks and their radio signals are used for range and/or direction measurement. Signals are identified by frequency and/or aspects of their modulation. Examples include GNSS, Loran and ultrawideband positioning systems.

Feature matching systems use features of the environment as landmarks. Examples include buildings, roads, field

(c) 2010 IEEE. Personal use of this material is permitted. Permission from IEEE must be obtained for all other uses, in any current or future media, including reprinting/republishing this material for advertising or promotional purposes, creating new collective works, for resale or redistribution to servers or lists, or reuse of any copyrighted component of this work in other works. 
boundaries and terrain height. Traditional "map and compass" navigation is an example of feature matching. Automated feature-matching systems must both match features to a database and determine the ranges to and/or directions of those features. Feature-matching sensors include optical and infra-red imaging sensors, laser scanners and radar.

Signals of opportunity is a generic term for radio signals that may be used for positioning, but are not specifically designed or modified for that purpose. These will typically be broadcasting or communications signals. Note that, by convention, signals already used by the user equipment for communications purposes are not classed as signals of opportunity. A key benefit of using signals of opportunity is cost as there is no need to install additional transmission infrastructure. A further benefit, for military applications, is that signals that could identify the presence of a military operation are not required. However, a major drawback is that the transmissions will not have been optimised for positioning. For example, transmitters will typically not be time synchronised and the signal modulation will not have been optimised for ranging accuracy.

Television and radio broadcasts are often used as signals of opportunity because the modulation format of these signals is publicly known and transmitter locations (to a few tens of meters) are publicly available. In recent years, positioning using both digital television [6, 7] and digital audio broadcasting (DAB) [8] signals has been demonstrated.

This paper focuses on amplitude modulation (AM) radio broadcasts in the medium frequency (MF) band. As there is no time synchronisation between AM radio broadcasts, any positioning system must be differential, comparing measurements made at a rover or mobile receiver at an unknown location with a reference or base receiver at a known location. Signals are captured simultaneously at the reference receiver and user locations. Signature parameters of the signals are then brought together for analysis to yield measurements of the time difference of arrival (TDOA) (between the two receivers) of each signal. These TDOA measurements can subsequently be processed to give the position of the user.

A wide range of $\mathrm{AM}$ radio broadcasts are currently available in most countries. In the MF band, the predominant ground-wave propagation mode offers better coverage in remote areas and over sea than higher frequency signals. Signals in these bands are also difficult to jam over large areas as high transmission powers are used to overcome relatively inefficient receiver antennas. However, not all locations are served by a sufficient number and geometry of AM radio broadcasts to generate a position solution. Therefore, a practical signals of opportunity positioning system would use AM signals alongside other signals, such as frequency modulation (FM) radio broadcasts, DAB and television. Alternatively, a hybrid positioning system, combining differential AM ranging with differential Loran could be implemented. This would be useful in areas where there are insufficient Loran signals to meet the accuracy and integrity requirements stand-alone.

AM radio positioning systems previously reported in the literature $[9,10,11]$ have used carrier phase measurements to generate a position solution. A sub- $25 \mathrm{~m}$ accuracy has been reported for such systems under optimum conditions. However, there are problems. Range derived from carrier phase has an ambiguity equal to the wavelength of the signal $(200-550 \mathrm{~m}$ in the MF broadcast band). To resolve this ambiguity, either the user must start at a known position [9] or a large number of signals must be receivable to enable consistency-based ambiguity resolution [10]. Furthermore, it is necessary to calibrate for azimuth-dependent phase biases at the transmitter and ground-wave propagation effects. However, the greatest limitation is that absorption and re-radiation of the signals produces large random phase variations indoors, in deep urban areas and near transmission lines [11]. Consequently, the derived position may suffer from unreliability and rapid deterioration in adverse environments.

\section{CONTEXT AND OVERVIEW}

This paper explores positioning using TDOA measurements obtained by correlating modulation information between the reference and user receivers over intervals of half a second. These measurements are unambiguous and are affected much less by re-radiation effects. However, they are much noisier than carrier-phase derived TDOA. The modulation-based TDOA measurements may be used in four different ways to form a navigation solution:

1. A snapshot position solution using only single-epoch modulation-based TDOA measurements. This is equivalent to a snapshot GNSS solution using differential code measurements only.

2. A snapshot position solution using several epochs of modulation-based TDOA measurements that have been smoothed using either time-differenced carrier-phase TDOA or differential Doppler shift. This is equivalent to a snapshot GNSS solution using differential carrier-smoothed code.

3. An extended Kalman filter (EKF)-based position solution using both modulation-based TDOA measurements and either time-differenced carrier-phase TDOA or differential Doppler shift measurements. This is equivalent to an EKF GNSS solution using both differential code and differential carrier measurements.

4. A snapshot position solution using carrier phase-based TDOA measurements with a modulation-based solution used to bound the ambiguity resolution search space, enabling the ambiguities to be resolved with fewer AM signals. This is equivalent to a real-time kinematic (RTK) differential carrier phase-based GNSS solution.

This paper focuses on the first option, using modulationbased TDOA measurements only. Investigations of the remaining methods will be described in future publications. These will need to incorporate integrity checks to identify phase or Doppler shift measurements corrupted by re-radiation effects and can use the modulation-only position solution as a reversionary mode.

The method presented here for obtaining range information by modulation correlation is potentially applicable to any signal with sufficient information contained within its 
modulation. It is not even necessary to know the modulation format of that signal provided it may be separated from its neighbors. Consequently, it forms the basis of a potential useanywhere heterogeneous-signal radio positioning system, which can opportunistically use whatever signals happen to be available at a particular location.

This paper is organized as follows. Section III introduces the concepts of the system, including a description of the system and an overview of the process of obtaining a position from the measurements. Section IV introduces the system design and explains the process of extracting timing information from the signals. Section V presents results obtained from simulations that have been designed to assess the quality of positioning that can be obtained from the AM MF broadcast band, across Great Britain. Section VI presents details of series of preliminary trials. This includes details of the demonstration apparatus and of the systems positioning performance during the trials. Conclusions and future work are presented in section VII.

\section{SYSTEM CONCEPT}

\section{A. System Description}

AM broadcast signals have modulation content that varies from signal to signal. As a result, any positioning system that uses modulation must be differential: comparing measurements made at a rover, at an unknown location, with a reference, at a known location.

A representation of the system is presented in Fig. 1. Shown in the figure is a reference receiver, $R$, and roving receiver, $A$, and a set of $N$ transmitters, $\left\{T_{1}, T_{2} \ldots T_{N}\right\}$. Also shown in the diagram is a transmission channel that allows information transfer between the reference and the rover.

The AM transmitters emit a set $n$ of signals that have the potential to be used for position - signals that shall be referred to as candidate signals. It is commonplace for more than one AM transmission to originate from the same transmitter site. Therefore the number of candidate signals tends to be greater than the corresponding number of transmitter sites (i.e. in general $n>N)$.

Only one mobile receiver and one reference receiver is shown in the figure. However, it is conceivable to extend the system to incorporate any number of rover and reference stations. Moreover, it is possible to blur the distinction in roles of the two stations. For example, stations could offer dual reference/rover functionality, as with network or relative positioning systems [5]. Without loss of generality, only a single rover and single reference receiver are considered in this paper.

Signals are captured simultaneously at the reference receiver, $R$, and rover, $A$. Signature parameters of the signals are then brought together for analysis to yield TDOA measurements and subsequently a position.

For real-time operation it is necessary to introduce a transmission channel, or datalink, between the base and rover receivers. The chosen direction of the datalink is largely application driven. For example, for tracking applications the system operator desires knowledge of the user, or participant. In this scenario it is possible to use a datalink that operates
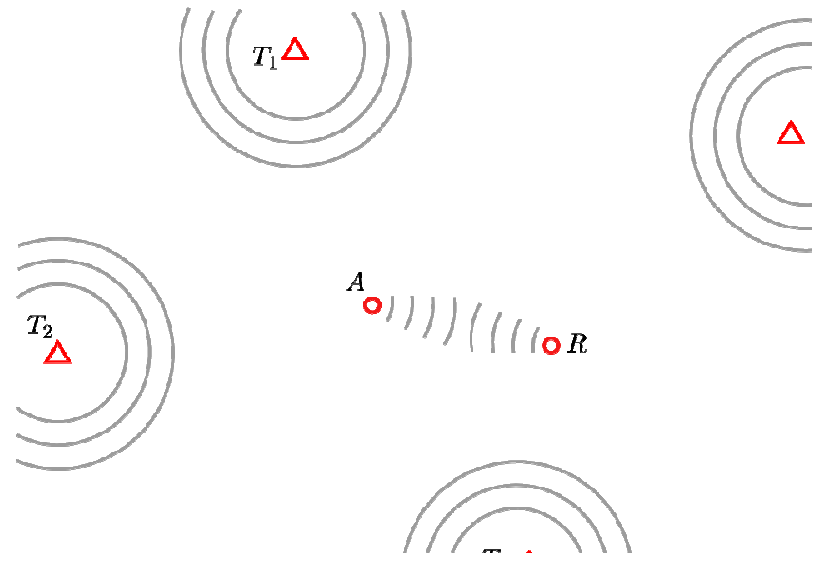

Figure 1. System Diagram

from the rover to reference receiver - subsequent analysis being performed at the latter. Alternatively, for navigation applications, a datalink from the reference to the rover can be used - subsequent analysis being performed by the user. This allows the system to operate with an unlimited number of participants. This mode of operation is also suitable for covert applications, where the user does not wish to reveal his or her location by radiating a signal.

\section{B. From Measurement to Position}

As explained in Section IV B, TDOA measurements are formed from a sub-set of the signals received by both the reference and rover - each signal producing one TDOA measurement. The TDOA measurements, together with the known transmitter and reference receiver locations, are used to determine the position of the mobile receiver.

Multiplying a TDOA measurement by the signal propagation speed (approximately the speed of light) gives a measurement of the difference in the pseudo-ranges from the transmitter to each of the two receivers. Adding the known range between the transmitter and the reference leaves a transmitter to rover pseudo-range analogous to that measured by GNSS user equipment [12]. The key difference here is that the rover receiver clock offset is with respect to the reference receiver clock instead of the transmitter clock.

The position and clock offset is determined from a set of pseudo-range measurements using an estimation algorithm and a measurement model (the deterministic part of which is sometimes known as a functional relationship). As groundwave propagation is dominant for MF broadcasting, the rover position can be determined using a measurement model similar to that used for Enhanced Long range navigation (ELoran) [5]. Note that vertical position information cannot be obtained from ground-wave propagated signals.

Where the baseline between the rover and reference is short, a Cartesian approximation may be used, enabling a version of the GNSS measurement model [5][13] with two 


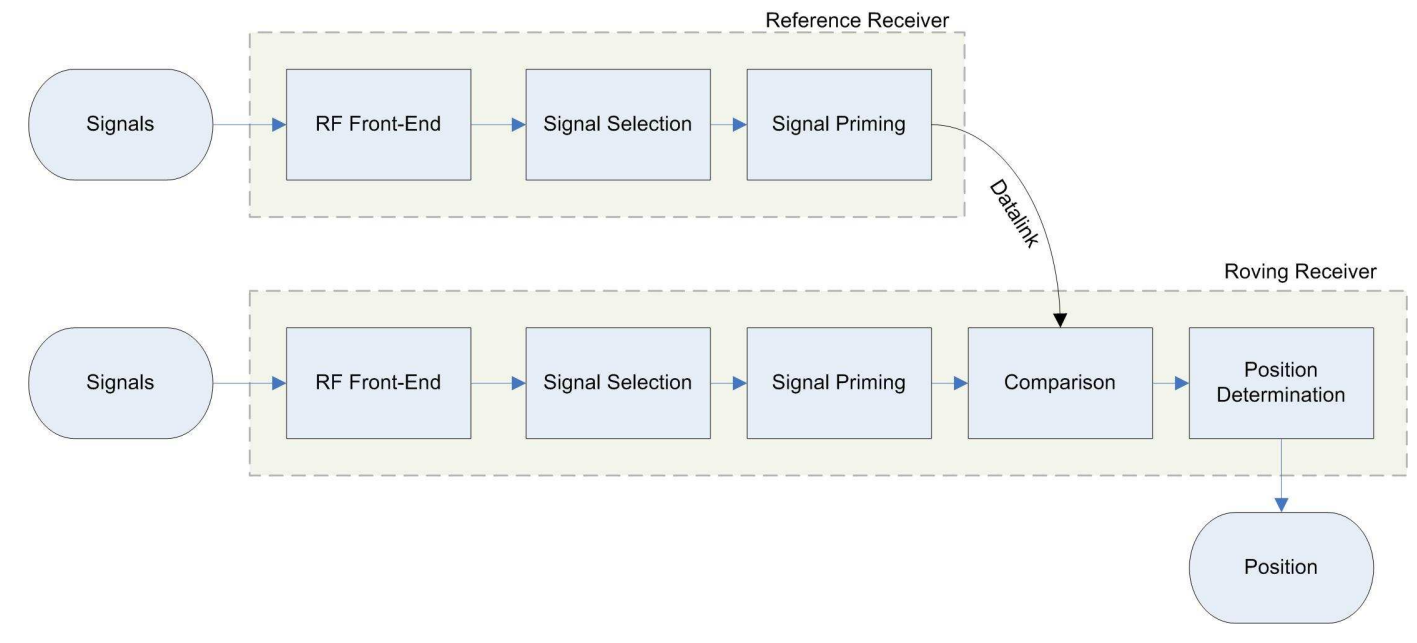

Figure 2. System Architecture

used for the work presented here. The relationship between the measurements and the rover position and clock offset is nonlinear, requiring the use of a nonlinear estimation algorithm. For the preliminary results presented here, an iterated least-squares (ILS) algorithm has been used with the reference receiver position used for initialization.

Once an initial position solution has been obtained, the measurement model may be linearized, enabling an extended Kalman filter to be used to process multiple-epoch TDOA measurements. However, this is a subject for future research.

In order to make TDOA measurements, the signal observation periods (or windows) at the base and mobile receivers must be synchronised. This is to ensure that the observation windows are approximately placed to observe the same portion of signal as it appears at two different locations. In principle, the receiver clock offset term in the position solution may be used to periodically resynchronize the rover receiver clock with that of the reference receiver in order to maintain alignment. However, initial time synchronization must be performed before a position solution is available. One option is to incorporate synchronization pulses in the data link between the rover and reference.

\section{SYSTEM DESIGN}

\section{A. System Architecture}

A differential Signal of Opportunity (SOP) system operates by simultaneously capturing multiple AM signals. In early SOP systems (see, for example, [9][14][15]) the process of simultaneous capture was accomplished by arranging a set of narrowband receivers in parallel. For these systems, each narrowband receiver was tuned to receive only one signal at any one time. In addition, reference and rovers were coordinated so that they were tuned to the same sets of signals. arrangement whereby a single wideband receiving apparatus is employed at both reference and rover. This receiving apparatus employs an analog to digital converter (ADC) to digitize the incoming AM MF broadcast band. Subsequent processing is achieved digitally. The wideband approach has several advantages over a narrowband approach. Most notably, a wideband system is capable of practically receiving a much larger number of signals at any one time.

Shown in Fig. 2 is a representation of the system architecture. Marked on the diagram are the reference and rover receivers. The two receivers are connected by a datalink that is used to send signal parameters from the reference receiver to the roving receiver. Signal comparison and position determination is accomplished at the roving receiver. Note that the three functional blocks of the reference receiver are mirrored in the roving receiver.

The first stage in each receiver is the Radio Frequency (RF) front end; this block comprises a wideband antenna, followed by analog signal conditioning components and finally an ADC. The ADC issues a digital snapshot of the entire spectrum.

The second stage in each receiver is the signal selection block. This selects a sub-set of signals from the candidate signal set. This procedure currently relies on an approximate apriori position prediction. The a-priori position is used to query an almanac database that contains a list of signals that offer good positioning potential for that particular location. The almanac database has been developed by considering a combination of factors, such as propagation loss, the extent of cross channel interference and signal geometry, all of which are used to assess the eligibility of a signal for positioning. Additionally, the almanac database is needed to match a received signal to its transmitter source - information that is required by the positioning algorithm. Following selection, the chosen signals are isolated from their neighbors by band-pass filtering. The isolated signals are then down-converted and 
non-coherently demodulated. What remains is a set of baseband audio signals.

The signal selection block is followed by the signal priming block. The signal priming operation prepares the signals for transmission across a datalink. This is achieved by applying a threshold to the signals in the frequency domain, to achieve, what is often referred to as, a sparse representation. This procedure is discussed further in section IV $\mathrm{B}$.

The comparison block cross-correlates signal pairs. Specifically, it takes the rover receiver signals and correlates them with counterpart reference receiver signals. The output from this block is a set of TDOA measurements corresponding to the signal pairs. The position determination block employs the set of TDOA estimates, together with the known transmitter locations, to solve for position.

\section{B. Extraction of Timing Information}

For the system described, it is necessary to measure the time difference of arrival (TDOA) of two signals. As described in section III B, the TDOA, is the difference in arrival times, according to local clocks, of a signal measured at reference and rover locations.

For AM broadcast signals, one possibility for obtaining TDOA measurements involves measuring the carrier phase of the two signals. The carrier phase measurements are then differenced and multiplied by the common frequency of the two signals to arrive at a TDOA measurement. However, the resulting measurement differs from the desired TDOA measurement by an integer number of periods of the measured carrier signal. In order to solve for position it is necessary to determine this unknown number of periods: a process often referred to as ambiguity resolution. For a system that uses AM signals it has been demonstrated [10][11] that, in some environments, noise and re-radiation effects render ambiguity resolution impossible. Moreover, incorrect resolution results in a position fix that is often many kilometers away from the true position [10][11].

Another possibility for obtaining TDOA measurements involves comparing the modulation of two signals. Unlike carrier phase measurements, the resulting measurements are un-ambiguous. This allows a position fix to be obtained without reliance on ambiguity resolution. As a result, a system of this form can be expected to be more robust, and less susceptible to deterioration in performance in adverse environments. However, there is a cost. An AM broadcast signal is formed by modulating a baseband audio signal onto a radio frequency carrier. The envelope of the resulting signal takes the same form as the modulating audio signal. Even the highest audio frequencies contained on the envelope are approximately 1000 times less than the carrier frequency. This makes it much more challenging to obtain high accuracy TDOA measurements from the modulation than from the carrier - even though the measurements aren't subject to ambiguity.

The system presented in this paper obtains TDOA measurements from the modulation content. The modulated signals are brought together and compared using a technique called generalized cross correlation (GCC). GCC is a method that was proposed by Knapp and Carter in 1976 [16]. The technique involves the application of a pre-filter prior to cross correlation. The pre-filter is designed to improve the TDOA estimate, in general, by amplifying the regions of the signal spectrum that has high signal to noise ratio, whilst attenuating the regions which have low signal to noise ratio [17].

The technique that has been used to implement GCC first requires spectral estimation. Spectral estimation is used to deliver frequency domain representations of the signals. In particular, an estimate is made of the cross power spectral density (CPSD) function. This function is formed by taking the estimated frequency spectrum of one of the signals and multiplying it by the complex conjugate of the estimated frequency spectrum from the other signal (see, for example [18][19][20]).

Following spectral estimation, a prefilter is applied by multiplying the CPSD function by a weighting function. The weighted CPSD function is converted to the time domain by performing an inverse Fourier transform. The resulting function is an estimate of the cross correlation function, the maximum of which corresponds to the estimated value of the TDOA.

In order to achieved sub-sample precision it is necessary to interpolate between the samples of the cross correlation function. This is achieved using a using a technique based on analogue reconstruction theory, as described in [21]. Following reconstruction a brute force search algorithm locates the position of the peak, to obtain an estimate of the TDOA.

The performance of GCC is greatly affected by the choice of prefilter. Examples of common prefilters are smoothed coherence transform (SCOT) and Hannon-Thompson (HT) filter and the phase transform (PHAT) [17]. The appropriate choice of pre-filter depends on the properties of the transmission channel between the source of the signal and the receivers. In general, the SCOT filter performs well when there is strong tonal, or narrow-bandwidth, interference. A HT filter, has been shown to be the maximum likelihood estimator for a signal in the presence of white noise. The PHAT filter has been shown to perform well in high signal-to-noise conditions [17].

For AM broadcast signals, the optimum choice of filter is subject to further research. However, preliminary investigations indicate that the PHAT pre-filter performs particularly well for AM broadcast signals that are received with the highest signal strength. Whereas, for signals with relatively low signal strength, the HT filter performs well.

In addition to the choice of pre-filter, preliminary research indicates that the content of the modulation greatly affects the accuracy of TDOA estimate. For example, when the spectrum of the modulation is flat and of low received power the accuracy of the TDOA estimate tends to be extremely poor. Whereas, when the spectrum of the modulation is of high received power with pronounced peaks the accuracy of the TDOA estimate tends be at its highest. 


\section{POSITIONING QUALITY ASSESSMENT}

Assessing the positioning quality of AM broadcast signals is motivated for two reasons: 1) to predict suitability and likely performance of the system 2) to assist in the a-priori selection of a set of signals for correlation.

Great Britain is in International Telecommunications Union (ITU) region 1. In this region the medium frequency section of the spectrum used for AM broadcasting spans from $531 \mathrm{kHz}$ to $1602 \mathrm{kHz}$. It is split into 120 uniformly spaced channels of 9 $\mathrm{kHz}$. However, because of channel re-use, there are many more stations than there are channels.

Shown in Fig. 3 and Fig. 4 are plots of the coverage, according to a simple model, of AM MF broadcast signals across Great Britain. Fig. 3 shows a prediction of the number of signals that can be received at any one location. In the population-dense southern region of Great Britain the number of signals received approaches its maximum of approximately thirty, whereas in the sparsely populated northern region, signal reception drops to a minimum of three signals. Also shown on the plot are the transmitter locations (marked with triangles). Incidentally, there are 94 transmitter sites and 232 broadcasts [22][23].

Shown in Fig. 4 is a plot of the Horizontal Dilulution of Precision (HDOP) across Great Britain. HDOP is a measure of the geometry-dependent effects on precision [5][13]. In the context of this system, it is the ratio of the TDOA variance for the set of chosen signals to the root mean square of the horizontal position error. The HDOP for this chart was constructed for a system that used all available candidate

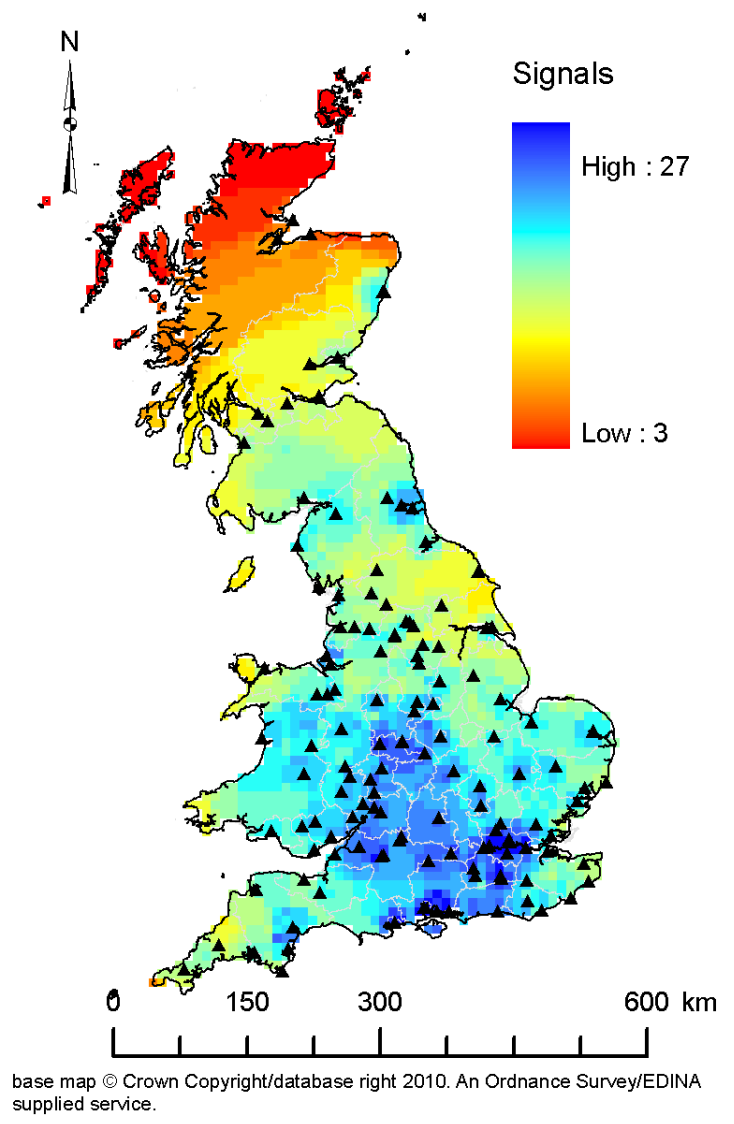

Figure 3. Number of signals received across Great Britain signals for positioning.

The coverage model used to construct the plots shown in Fig. 3 and Fig. 4 is based on a simple propagation formula [24][25]. It should be noted that the model makes many simplifying assumption. For example, it has been assumed that the terrain is flat with a homogenous set of electrical parameters. Moreover, it has been assumed that transmitters issue an azimuthally isotropic signal. It should be appreciated that some of the AM transmissions included are purposefully directional. In addition, the model fails to account for crosschannel interference, which has the potential to render a signal unusable for positioning. Cross-channel interference is particularly pronounced on channels that are used within the United Kingdom for the transmission of single frequency networks (SFN). Additional, potentially important, un-modeled effects include urban fading and sky wave interference.

The model assumes a reception threshold. The reception threshold is the cut-off received power level below which the signals are considered unusable. An estimation of this cut-off level has been made using empirical data obtained from the demonstration apparatus. In using empirical data, it is aimed to accommodate for antenna and front end losses that might occur in addition to propagation losses. Details of the demonstration apparatus are presented later in this paper.

\section{PRELIMINARY TRIALS}

\section{A. Demonstration System}

A demonstration system has been developed to prove the

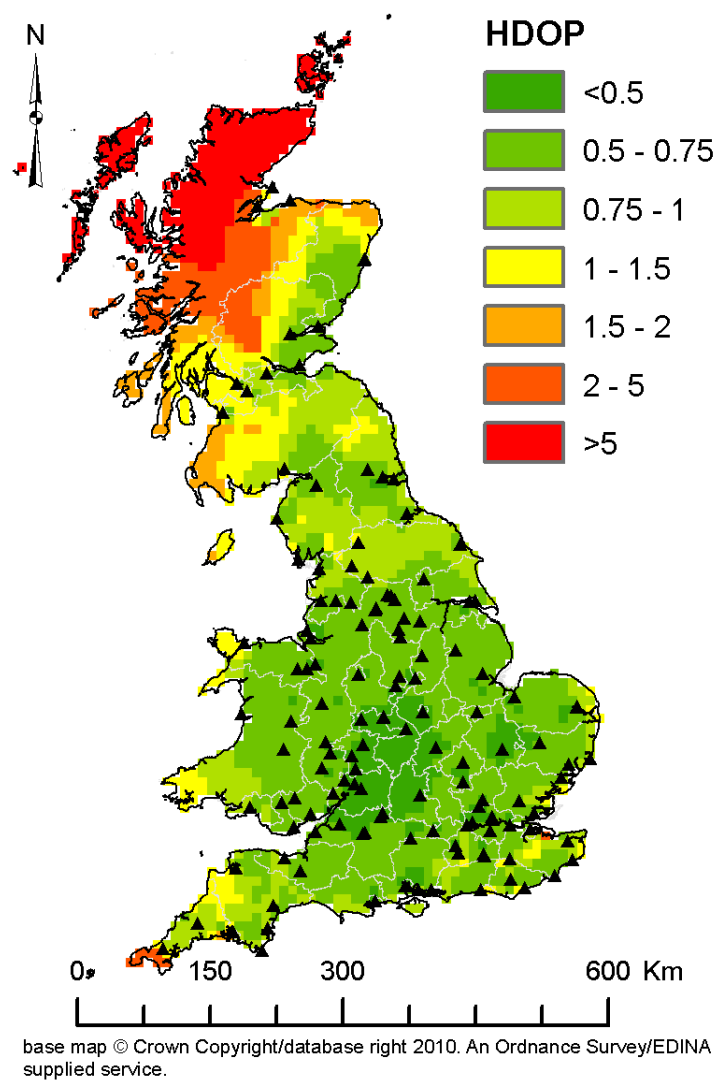

Figure 4. Simulated HDOP across Great Britain 
concept of AM positioning and predict the performance of a real-time system. In a similar manner to the system presented in sections III and IV, the demonstration system comprises of reference and rover receivers. Both receivers include an RF front end that is used to obtain a digital snapshot of the spectrum. Subsequent processing is then carried out digitally.

To allow real-time operation the system presented in sections III and IV incorporates a datalink. However, to ease implementation, the demonstration system has been designed to operate without a datalink. Instead, the digitized snapshot is stored in memory modules by the reference and rover receivers. Following a trial exercise, the data on the memory modules is downloaded to a PC for further processing.

Due to the absence of a datalink, it is necessary for the demonstration system to employ a method of synchronizing the observation windows used for correlation that does not rely on the transmission of either clock corrections or synchronization pulses. This has been achieved by incorporating a Global Positioning System (GPS) receiver into each of the demonstration system receivers. Timing information derived from the GPS receiver is subsequently extracted and used for synchronization. It should be appreciated that this reliance on GPS is only a feature of the demonstration system and that the system proposed in sections III and IV can operate entirely the active high-frequency antenna). The apparatus comprises the following parts:

- An active high-frequency antenna (Watson) followed by a $1.9 \mathrm{MHz}$ low-pass filter.

- A 14-bit $140 \mathrm{MS} / \mathrm{s}$ analog-to-digital converter (Analog Devices AD9254 on an Altera HSMC data capture board) that is used to capture the RF signal.

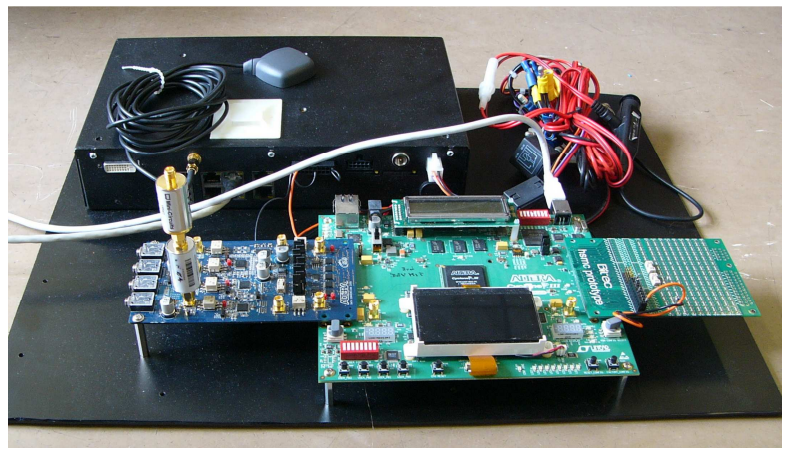

Figure 6. Photo of demonstration system apparatus

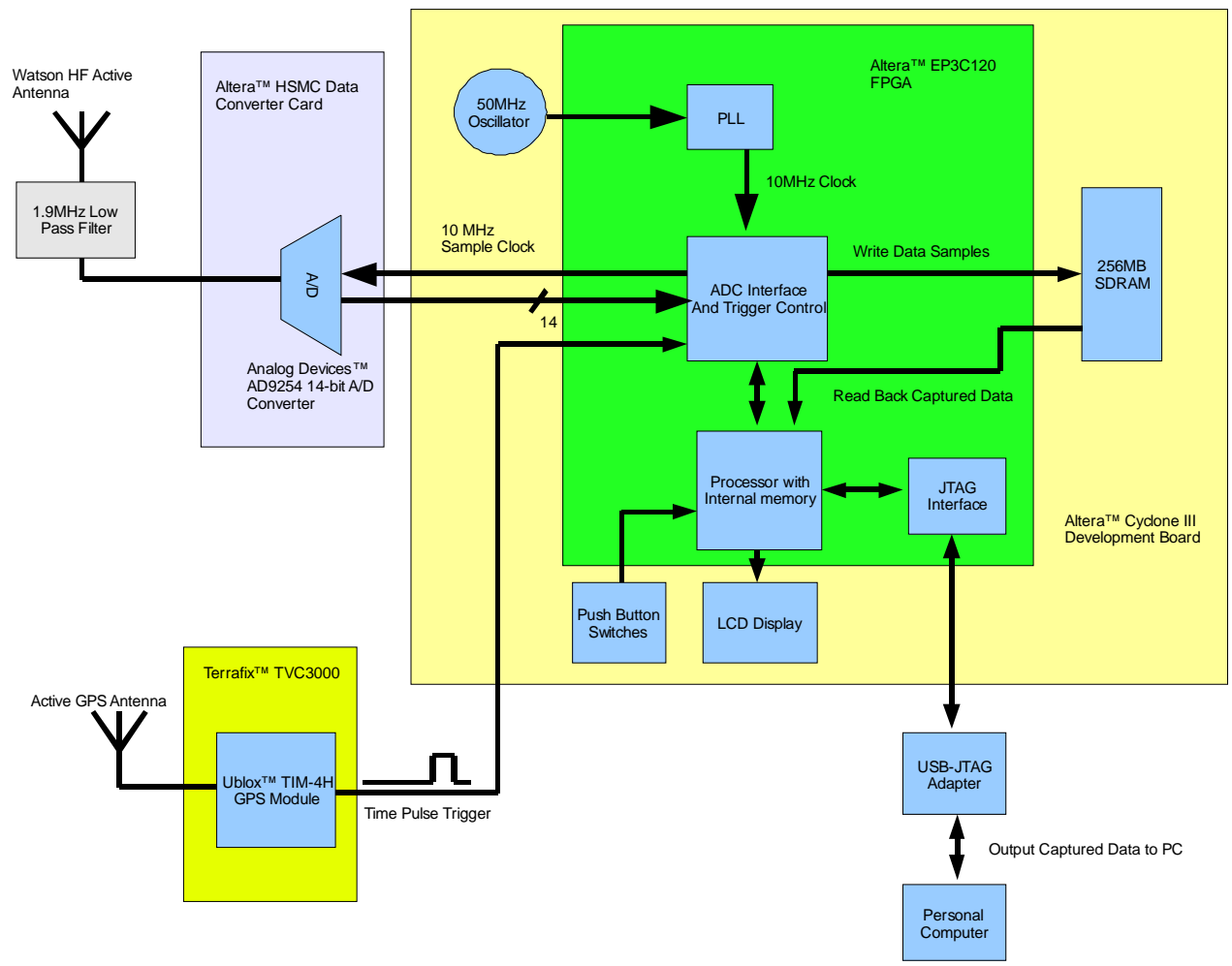

Figure 5. Schematic of demonstration system apparatus

independently of GPS.

Shown in Fig. 5 is a schematic of the demonstration system apparatus that is located at the reference and rover locations. Also, shown in Fig. 6 is a photo of the apparatus (not including
- Hardware that is implemented on a field programmable gate array (FPGA) development board (Altera Cyclone III 3C120 development board), that writes the ADC data samples to 
memory and then, once the capture is complete, writes the data to a personal computer via a Joint Test Action Group (JTAG) interface. A processor is instantiated within the FPGA that configures the capture hardware, provides user feedback via a liquid crystal display (LCD) and primes the capture system when a push-button is pressed, ready for capture synchronization via the GPS time pulse. The development board provides the $10 \mathrm{MHz}$ sample clock for the ADC.

- A GPS module residing in a Terrafix TVC3000 vehicle computer system, configured to produce a time pulse at the start of each minute, as defined by GPS time. The pulse is used to synchronize the capture of data at two different locations.

\section{B. Trial Experiments}

A series of preliminary trials have been conducted using the demonstration system described. The trials were conducted in an area located on the outskirts of the city of Stoke in the U.K. Presented in this paper are results from 5 trials. For each trial, a $0.5 \mathrm{~s}$ snapshot was used for position determination. Over the

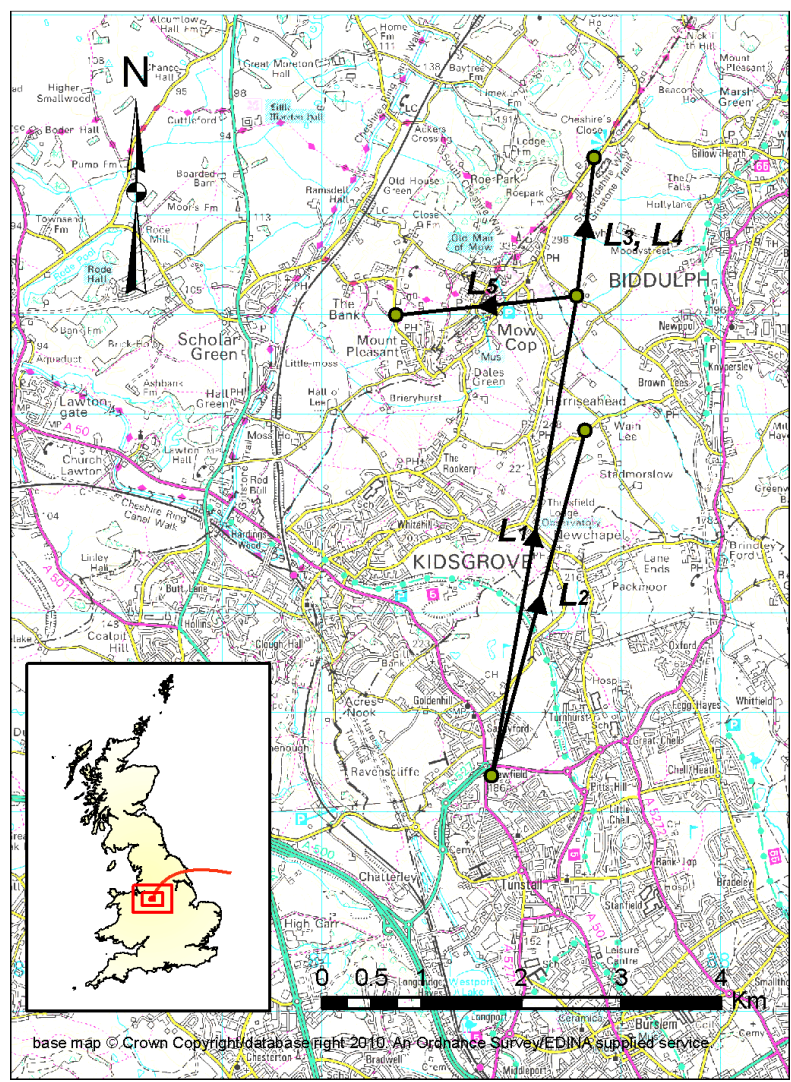

Figure 7. Preliminary trials test region

series of trials, the separation between the reference and rover in each trial varied from approximately $1.5 \mathrm{~km}$ to $5 \mathrm{~km}$.
Displayed in Fig. 7 is a map of the test region. The land use over the region is a mixture of urban and rural. Across the region, the terrain height varies by up to fifty meters. Marked on the map are the positions of the reference and rover receiver pairs and their corresponding hypothetical connecting lines (i.e. their baselines). The arrows on the baselines are drawn from the receiver that has been designated as the reference to the receiver that has been designated as the rover. The baseline of $i^{\text {th }}$ trial is denoted by $L_{i}$. Note that two of the trials, trials 3 and 4 , share a common baseline.

Shown in Fig. 8 and Fig. 9 are photos of trial locations. In the photos the white elongated cylinder contains the active antenna that is part of the demonstration system apparatus. Fig. 8 is the location used for base receiver in trials 1 and trials 2. The location is on the top floor of a brick building which has some cast iron supporting structure. Fig. 9 is representative of all other receiver locations that were used. These locations were outside in regions of mixed urban-agricultural land use.

For the five trials, a GPS fix of the receiver locations was obtained from the module that was incorporated into the demonstration apparatus. The GPS receiver positions were used to: 1) provide a 'known' location of the reference receiver - as required and by the position algorithm; 2) provide a location for the rover that

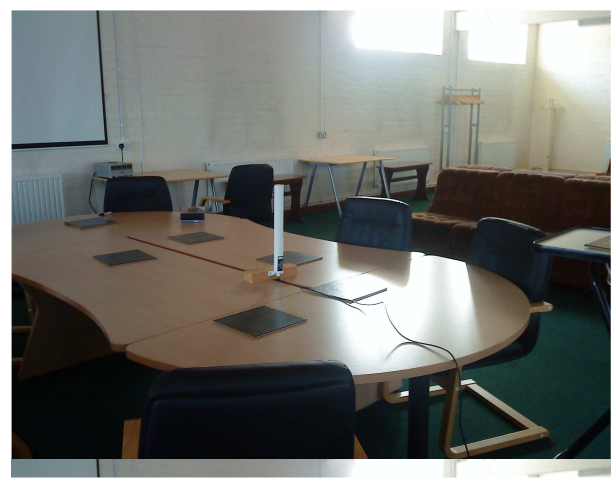

Figure 8. Reference receiver location used in trials 1 and 2

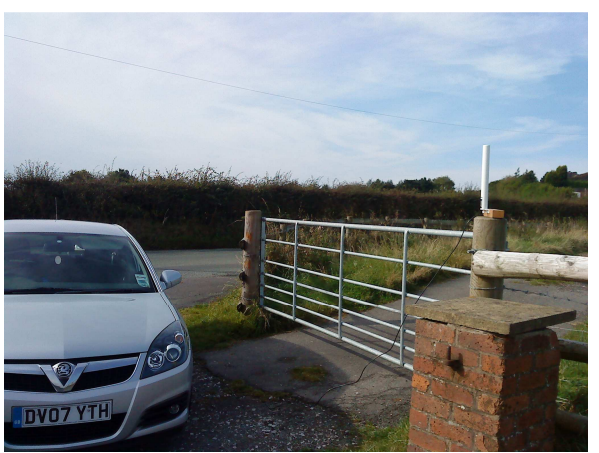

Figure 9. Representative of all receiver locations, with the exception of the reference receiver locations used in trials 1 and 2 .

could be compared to the SOP derived fix. The GPS receiver used was consumer grade and had an accuracy of approximately ten meters. 
Fig. 10 displays the difference between GPS-derived positions and SOP-derived position for the five fixes. Of interest is that the position offset between the two fixes was always towards the west. It is possible that this westward trend was caused by a bias in one or more of the TDOA measurements. The TDOA measurements could be biased as a result of cross-channel interference or the use of incorrect transmitter locations, however this is subject to further research.

Further details of the trials are presented in Table 1. Different sets of signals were used for the trials. The number of signals ranged from 6 to 9 . Due to transmitter site sharing, for all trials, the number of transmitter sites was less than the number of signals. The root mean square (RMS) of the position error was $321 \mathrm{~m}$.

TABLE I. TRIAL RESUlts

\begin{tabular}{|l|l|c|c|c|c|c|c|}
\hline \multirow{2}{*}{ Trial } & \multirow{2}{*}{$\boldsymbol{L} / \mathbf{m}$} & \multirow{2}{*}{$N_{S}$} & \multirow{2}{*}{$\boldsymbol{N}_{\boldsymbol{T}}$} & \multirow{2}{*}{ HDOP } & \multicolumn{3}{|c|}{ Position Error/m } \\
\cline { 5 - 8 } & & & & & East & North & Dist \\
\hline 1 & 4898 & 8 & 5 & 0.8584 & -11.8 & 26.2 & 28.7 \\
\hline 2 & 3599 & 8 & 4 & 1.0092 & -122.1 & -180.5 & 217.9 \\
\hline 3 & 1397 & 6 & 4 & 1.1161 & -241.8 & 158.4 & 289.0 \\
\hline 4 & 1397 & 9 & 5 & 0.8558 & -442.0 & 387.5 & 587.8 \\
\hline 5 & 2540 & 6 & 4 & 1.7346 & -194.1 & -147.1 & 243.6 \\
\hline
\end{tabular}

$L$ : baseline length; $N_{s}$ : number of signals; $N_{T}$ : number of transmission sites

\section{CONCLUSION AND FUTURE WORK}

The results from five preliminary trials demonstrated that it is possible to obtain a position solution accurate to a few hundred meters using the modulation content of AM broadcast signals. GCC has been shown to be an effective method of obtaining a TDOA measurement. Further, a position fix was obtained even when one of the receivers was located inside a brick building. This indicates that the system achieves a robustness that cannot be achieved for systems that also operate on the AM broadcast band but use phase positioning alone [10][11].

Multiple areas for further research to improve the position accuracy have been identified. Ranging errors will be investigated using further trials data in order to try and quantify the bias-like and noise-like components. The impact of modulation content (for example, speech versus music), signal to noise level, terrain, and reception environment on ranging errors will be studied. Furthermore, the relationship between the sharpness of the correlation peak and the ranging errors will be investigated. This should enable the various TDOA measurements within the position algorithm to be weighted according to their accuracy. Outlier detection will be implemented to minimize the impact of erroneous TDOA measurements on the position solution.

Improvements in performance can also be expected by careful selection of the optimal pre-filter, and signal segment

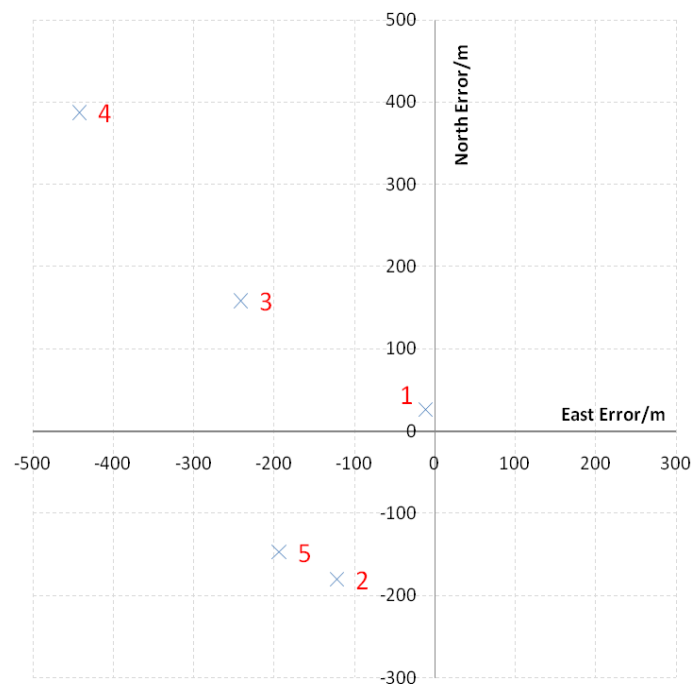

Figure 10. Position error for five trials

used for correlation. Additionally, the system performance is likely to improve by improving signal coverage modeling, so that the optimal subset of signals can be selected.

Positioning methods that combine modulation and carrierbased TDOA measurements, as described in Section II, will be assessed with the aim of obtaining a more accurate position solution.

Finally, the modulation correlation-based ranging method will be extended to work with generic signals, regardless of how the signal is modulated or even whether the modulation format is known. The first step will be to modify the correlation algorithms to use modulated signals, thus, eliminating the amplitude demodulation step. Following this, tests will be conducted using signals outside the AM broadcasting band.

\section{ACKNOWLEDGMENT}

The authors would like to thank Joe Woodward and Robert McHarry for contributions that they have made to the work presented in this paper.

\section{REFERENCES}

[1] J. Carroll et al, "Vulnerability assessment of the transportation Infrastructure Relying on the Global Positioning System," John A. Volpe National Transportation Systems Center report for US Department of Transportation, 2001.

[2] D. Last, "GNSS - The Present Imperfect," Proc. GPS Jamming \& Interference - A Clear and Present Danger, Teddington, UK, February 2010.

[3] M. Knight, and A. Finn, "The Impact of Ionospheric Scintillations on GPS Performance," Proc. ION GPS, Kansas City, MO, September 1996, pp. 555-564.

[4] A. Cerruti, "Observed GPS and WAAS signal-to-noise degradation due to solar radio bursts," Proc. ION GNSS 2006, Fort Worth, TX, September 2006, pp. 1369-1376.

[5] P. D. Groves, Principles of GNSS, Inertial, and Multisensor Integrated Navigation Systems, Norwood, MA: Artech House, 2008. 
[6] M. Rabinowitz, and J. J. Spilker, Jr., "A New Positioning System UsingTelevision Synchronization Signals," IEEE Transactions on Broadcasting, Vol. 51, No. 1, 2005, pp. 51-61.

[7] P. Kovář, and F. Vejražka, "Multi System Navigation Receiver," Proc. IEEE/ION PLANS, Monterey, CA, May 2008, pp. 860-864.

[8] D. Palmer, et al., "Non-GNSS radio positioning using the digital audio broadcasting (DAB) signal," Proc. ION GNSS 2009, Savannah, GA, September 2009, pp. 864-872.

[9] P. J. Duffett-Smith, and G. Woan, "The CURSOR radio navigation and tracking system," Journal of Navigation, Vol. 45, No. 2, 1992, pp. 157165.

[10] T. D. Hall, C. C. Counselman, III, and P. Misra, "Instantaneous radiolocation using AM broadcast signals," Proc. ION NTM, Long Beach, CA, January 2001, pp. 93-99.

[11] T. D. Hall, Radiolocation using AM Broadcast Signals, PhD thesis, Cambridge, MA: Massachusetts Institute of Technology, September 2002.

[12] K. A. Fisher, J. F. Raquet, and M. Pachter, "Cooperative estimation algorithms using TDOA measurements," Lecture Notes in Economics and Mathematical Systems, Vol. 588 2007, pp. 57-66.

[13] P. Misra, and P. Enge, Global Positioning System Signals, Measurements, and Performance, 2nd ed., Lincoln, MA: Ganga-Jamuna Press, 2006.

[14] W. Connelly (1986), "Telenav: A precision navgation system based upon television reception," Journal of Navigation, 33, pp.109-122., 1986

[15] E. J. Dalabakis and H.D. Shearer, "Position fixing system utilizing plural commercial broadcast transmissions and having frequency correction," US Patent 4054880, 1977
[16] C. Knapp and G. Carter, "The generalized correlation method for estimation of time delay", Acoustics, Speech and Signal Processing, IEEE Transactions on, 24(4), pp 320-327, Aug 1976.

[17] Y. Gao, M.J. Brennan, and P.F. Joseph, "A comparison of time delay estimators for the detection of leak noise signals in plastic water distribution," Journal of Sound and Vibration, 292:552-570, 2006.

[18] J. S. Bendat and A. G. Piersol. Engineering applications of correlation and spectral analysis, 2nd ed., John Wiley and Sons, Inc., New York etc, 1993, pp. 293-294.

[19] A. Oppenheim, and R. Schafer. Digital Signal Processing. Prentice-Hall Inc., 1975.pp 554-555

[20] S. K. Mitra. Digital Signal Processing. McGraw-Hill, 2006

[21] I. Cespedes, Y. Huang, J. Ophir, and S. Spratt, "Methods for estimation of subsample time delays of digitized echo signals," Ultrasonic Imaging, 17, 1995.

[22] BBC, "Helping receiving TV and radio," 2010, [online]. Available: http://www.bbc.co.uk/reception/transmitters/radio/index.shtml, [accessed on Feb. 17, 2010]

[23] Ofcom, "Tech parameters," 2010, [online]. Available: http://www.ofcom.org.uk/radio/ifi/rbl/engineering/tech_parameters/ [accessed on Feb. 17, 2010]

[24] N. DeMinco., "Propagation prediction techniques and antenna modeling (150 to $1705 \mathrm{khz}$ ) for intelligent transportation systems (ITS) broadcast applications," Antennas and Propagation Magazine, IEEE, 42(4): pp. 9 34, aug 2000.

[25] J. Griffths, Radio Wave Propagation and Antennas. Prentice/Hall International,1987, pp. 33-38

(C) 2010 IEEE. Personal use of this material is permitted. Permission from IEEE must be obtained for all other uses, in any current or future media, including reprinting/republishing this material for advertising or promotional purposes, creating new collective works, for resale or redistribution to servers or lists, or reuse of any copyrighted component of this work in other works. 